\title{
Editorial \\ Crystalline Micro- and Nano-Materials for Medical and Other Biochemical Applications
}

\author{
Songwen Tan ${ }^{1, *} \mathbb{1}$, Chuanpin Chen ${ }^{1, *}$, Xuncai Chen ${ }^{2, *}$ and Wenhu Zhou ${ }^{1, *}$ \\ 1 Xiangya School of Pharmaceutical Sciences, Central South University, 932 Lushan South Road, Yuelu District, \\ Changsha 410083, China \\ 2 School of Forensic Medicine, South Medical University, Shatai Road 1838, Baiyun, Guangzhou 510515, China \\ * Correspondence: stan0309@alumni.sydney.edu.au (S.T.); ccpin2000@hotmail.com (C.C.); \\ xche3815@smu.edu.cn (X.C.); zhouwenhuyaoji@163.com (W.Z.)
}

check for updates

Citation: Tan, S.; Chen, C.; Chen, X.; Zhou, W. Crystalline Micro- and Nano-Materials for Medical and Other Biochemical Applications. Crystals 2021, 11, 1361. https:// doi.org/10.3390/cryst11111361

Received: 3 November 2021 Accepted: 5 November 2021 Published: 8 November 2021

Publisher's Note: MDPI stays neutral with regard to jurisdictional claims in published maps and institutional affiliations.

Copyright: (c) 2021 by the authors. Licensee MDPI, Basel, Switzerland. This article is an open access article distributed under the terms and conditions of the Creative Commons Attribution (CC BY) license (https:/ / creativecommons.org/licenses/by/ $4.0 /)$.
The Special Issue on "Crystalline Micro- and Nano-Materials for Medical and Other Biochemical Applications" is a collection of seven original articles (including three research papers and four review papers) dedicated to theoretical and experimental research work that provides new insights and practical findings in the field of crystal-related biomedicine.

The use of crystalline micro- and nano-materials, based on organic and inorganic molecules, is a rapidly progressing field of medical and biochemical applications. Particular focus is given to solids with functional biochemical properties, which can be used in medical, pharmaceutical, environmental, microbial, energy, and many other biochemical applications. Although the synthesis of crystalline micro- and nano-materials has been largely carried out in recent years, the applications of these materials still face problems in different fields in practice.

On the topic of crystalline micro- and nano-materials for medical and biochemical applications, it is important to focus more on the application aspect of these materials in the near future, to aid the development of this discipline. First of all, the fundamental theories of the synthesis methods need to be revealed to control the crystallization process and other engineering aspects. Studies need to be performed at molecular, cellular, and higher levels with the well-designed materials, to provide access to the materials in medical and biomedical applications. In vivo and in vitro studies, and higher-level clinical studies, are required in order for these materials to be used on the market. Mathematical modeling may be performed to improve the technology. In addition, ethical research and social science studies assist the development of these crystalline micro- and nano-materials for medical and biochemical applications.

Here, we outline theoretical work in which crystalline micro- and nano-materials were evaluated for medical and biochemical applications. Xiang et al. [1] tailored an $\alpha / \beta$ ratio of pollen-like anhydrous lactose with an anti-solvent technique. The products can be used as ingredient carriers to improve the solubility of pharmaceuticals. The $\alpha / \beta$ crystalline state played an important role in the release control of the crystalline micro- and nano-materials. Guo et al. [2] coated dopamine-grafted hyaluronic acid on hyperbranched poly( $\beta$-amino esters)/DNA nano-complexes for enhanced gene delivery and biosafety. The vector has good transfection efficiency and excellent biocompatibility, and can be used for gene delivery applications. Zhong et al. [3] coated hyaluronic acid on MTX-PEI nanoparticles for targeted rheumatoid arthritis therapy. The MTX-PEI@HA NPs were a promising MTX-based nanoplatform for the treatment of RA. The two articles based on material coating studied the crystalline biomaterials and their applications.

The second group of papers reviews the current techniques for the use of crystalline micro- and nano-materials in medical and biochemical applications. In this field, Yi et al. [4] concluded the existing photocatalytic materials used to treat cancer, as well as the current challenges in the application of cancer therapy. Advanced crystalline micro- and nanomaterials have been used in up-to-date biomedicines. Tan et al. [5] reported the possible 
preparation approaches of nano-cocrystals. The review work provides guidance to develop new nano-cocrystals with commercial value in the pharmaceutical industry. Liu et al. [6] concluded the common reactive oxygen species (ROS) nanomaterials and reviewed the utilization of ROS in dentistry, highlighting the potential applications and safety of these materials in clinical treatment. The proposed future prospect of these materials is their use as a clinic dental cure. Li et al. [7] summarized the current studies of MALAT1 in the fibrosis of various organs. This review contributed to better understanding the molecular mechanism of fibrosis and the potential of MALAT1 as a novel therapeutic target for fibrosis. These review papers have comprehensively reported the synthesis, mechanisms, and applications of crystalline biomaterials.

I hope that this collection of papers will meet the expectations of readers looking for new advances in the field of crystalline micro- and nano-materials, as well as bringing inspirations for future research work on the medical and biochemical applications of these materials.

Conflicts of Interest: The authors declare no conflict of interest.

\section{References}

1. Xiang, J.; Wang, B.; Fu, L.; Chen, C.; Liu, W.; Tan, S. Tailoring $\alpha / \beta$ Ratio of Pollen-Like Anhydrous Lactose as Ingredient Carriers for Controlled Dissolution Rate. Crystals 2021, 11, 1049. [CrossRef]

2. Guo, M.; Meng, Y.; Qin, X.; Zhou, W. Dopamine-Grafted Hyaluronic Acid Coated Hyperbranched Poly( $\beta$-Amino Esters)/DNA Nano-Complexes for Enhanced Gene Delivery and Biosafety. Crystals 2021, 11, 347. [CrossRef]

3. Zhong, S.; Liu, P.; Ding, J.; Zhou, W. Hyaluronic Acid-Coated MTX-PEI Nanoparticles for Targeted Rheumatoid Arthritis Therapy. Crystals 2021, 11, 321. [CrossRef]

4. Yi, H.; Cheng, Z. A Literature Review on High-Performance Photocatalysts for Sustainable Cancer Therapy. Crystals 2021, 11, 1241. [CrossRef]

5. Tan, J.; Liu, J.; Ran, L. A Review of Pharmaceutical Nano-Cocrystals: A Novel Strategy to Improve the Chemical and Physical Properties for Poorly Soluble Drugs. Crystals 2021, 11, 463. [CrossRef]

6. Liu, F.; Hong, T.; Xie, J.; Zhan, X.; Wang, Y. Application of Reactive Oxygen Species-Based Nanomaterials in Dentistry: A Review. Crystals 2021, 11, 266. [CrossRef]

7. Li, Y.; Liu, F.; Cai, Y.; Yang, Y.; Wang, Y. LncRNA MALAT1: A Potential Fibrosis Biomarker and Therapeutic Target. Crystals 2021, 11, 249. [CrossRef] 